\title{
Intermolecular interactions in solid benzene
}

\author{
G. J. Kearley ${ }^{\text {a) }}$ \\ Department of Radiation, Radionuclides and Reactors, Faculty of Applied Sciences, Delft University of \\ Technology, Mekelweg 15, 2629 JB Delft, The Netherlands \\ M. R. Johnson \\ Institut Laue-Langevin (ILL), BP 156, 38042 Grenoble, Cedex 9, France \\ J. Tomkinson \\ CCLRC, The ISIS Facility, Rutherford Appleton Laboratory, Chilton, OX fordshire OX11 OQX, United \\ Kingdom
}

(Received 25 August 2005; accepted 8 November 2005; published online 30 January 2006)

\begin{abstract}
The lattice dynamics and molecular vibrations of benzene and deuterated benzene crystals are calculated from force constants derived from density-functional theory (DFT) calculations and compared with measured inelastic neutron-scattering spectra. A very small change $(0.5 \%)$ in lattice parameter is required to obtain real lattice-mode frequencies across the Brillouin zone. There is a strong coupling between wagging and breathing modes away from the zone center. This coupling and sensitivity to cell size arises from two basic interactions. Firstly, comparatively strong interactions that hold the benzene molecules together in layers. These include an intermolecular interaction in which $\mathrm{H}$ atoms of one molecule link to the center of the aromatic ring of a neighboring molecule. The layers are held to each other by weaker interactions, which also have components that hold molecules together within a layer. Small changes in the lattice parameters change this second type of interaction and account for the changes to the lattice dynamics. The calculations also reveal a small auxetic effect in that elongation of the crystal along the $b$ axis leads to an increase in internal pressure in the $a c$ plane, that is, elongation in the $b$ direction induces expansion in the $a$ and $c$ directions. () 2006 American Institute of Physics. [DOI: 10.1063/1.2145926]
\end{abstract}

\section{INTRODUCTION}

Benzene is generally regarded as the prototypical example of an aromatic molecule and in the solid state it provides the simplest real system in which interactions between aromatic molecules can be studied. The aim of the present work is to understand the intermolecular interactions at a microscopic level that lead to molecular packing, lattice dynamics, phase behavior, and ultimately the possibility of new bulk properties in aromatic systems. Over the years there have been a number of studies that are pertinent to this work: crystal structure, ${ }^{1}$ phases, ${ }^{2,3}$ and vibrational spectra, ${ }^{4-9}$ from both the experimental and theoretical standpoints.

The present work is mainly concerned with the calculation of intermolecular interactions using density-functional theory (DFT) methods, but in order to connect with experiment we compare our calculations not only with existing crystallographic data and optical vibrational spectroscopies but also with new inelastic neutron-scattering (INS) spectroscopy data. In this way we establish an almost parameter-free model that is capable of reproducing the static and dynamic structure factors. The use of DFT methods for periodic systems for the determination of molecular vibrations and zonecenter $(\Gamma$ point) lattice modes, and comparison of these with INS spectra, has become common place in recent years. ${ }^{10,11}$ Here these methods are extended to full lattice dynamics calculations, taking into account the whole Brillouin zone.

${ }^{a)}$ Electronic mail: g.j.kearley@tnw.tudel
Increasingly, these calculations are being used to simulate not only the coherent INS spectra from single crystals but also the incoherent INS spectra of powdered samples. ${ }^{12-14}$ The latter is a far more straightforward experimental technique and alleviates the need for large single crystals of deuterated materials in the study of lattice dynamics.

Having established a model that reproduces the experimental data we shall exploit it to discern three major intermolecular interactions that hold the crystal together. One of these interactions is between the $\mathrm{H}$ atom of one molecule with the center of the aromatic ring of a neighboring molecule. Clearly, this is unique to aromatic systems and it is important to establish the relative strength of this interaction by comparing simulation and experiment, mainly lattice dynamics in this case, and by investigating the effects of uniaxial and isotropic pressures in the simulation.

\section{EXPERIMENT}

Benzene and deuterated benzene were obtained from The Aldrich Chemical Company and used without further purification. Samples were loaded in aluminum sample containers and cooled to $15 \mathrm{~K}$ using a standard cryostat. Data were collected using the now defunct TFXA spectrometer (replaced with TOSCA) (Ref. 15) at the ISIS pulsed neutron facility in the UK. Raw data were transformed into $S(\mathbf{Q} \omega)$ using standard algorithms. 


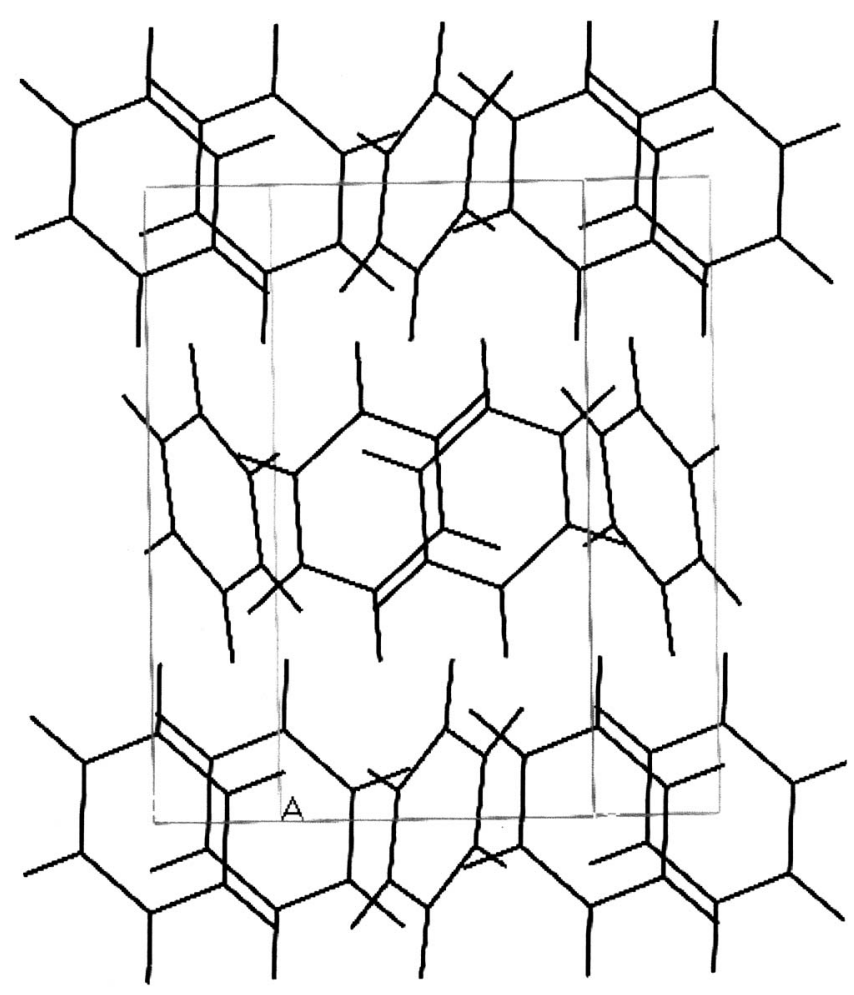

FIG. 1. Schematic illustration of the crystal structure of $\mathrm{C}_{6} \mathrm{D}_{6}$ from Ref. 1 showing the relative orientation of molecules within the layers. The long $b$ axis is vertical.

\section{CRYSTALLOGRAPHIC INFORMATION}

In a DFT calculation of solid-state structure and dynamics of a molecular crystal, the only input is the measured crystal structure. For benzene, the structure has been determined at $4.2 \mathrm{~K}$ to be orthorhombic in the space-group $\mathrm{Pbca}$, the unit cell and its contents being illustrated in Fig. 1. The cell parameters are $a=7.355, b=9.371$, and $c=6.700 \AA .{ }^{1}$

\section{COMPUTATIONAL METHODS}

Energy calculations and structural optimization were made using VASP $4.5,{ }^{16}$ using the PBE exchange-correlation functional and PAW pseudopotentials with an energy cutoff of $600 \mathrm{eV}$. A single crystallographic unit cell was used for all calculations, with the reciprocal lattice being sampled using eight $k$ points $(=[2,2,2])$. Single-point energy calculations were made for a series of structures in which the crystallographically distinct atoms were displaced by $0.03 \AA$ in positive and negative directions along the $x, y$, and $z$ directions. These calculations gave the Hellmann-Feynmann (HF) forces acting on each atom and were used as input for the lattice-dynamics program PHONON4.2.4. ${ }^{17}$ Nonzero force constants were determined using the single unit cell, and it was found that all of these decayed by more than three orders of magnitude in going from the cell center to the nearest cell boundary. Phonon was used to calculate the eigenfrequencies, dispersion curves, and simulated inelastic neutronscattering spectra $S(\mathbf{Q} \omega)$. The same HF forces were used for both $\mathrm{C}_{6} \mathrm{H}_{6}$ and $\mathrm{C}_{6} \mathrm{D}_{6}$, but the appropriate atomic masses and scattering cross sections were used in the lattice-dynamics calculations and INS calculations.

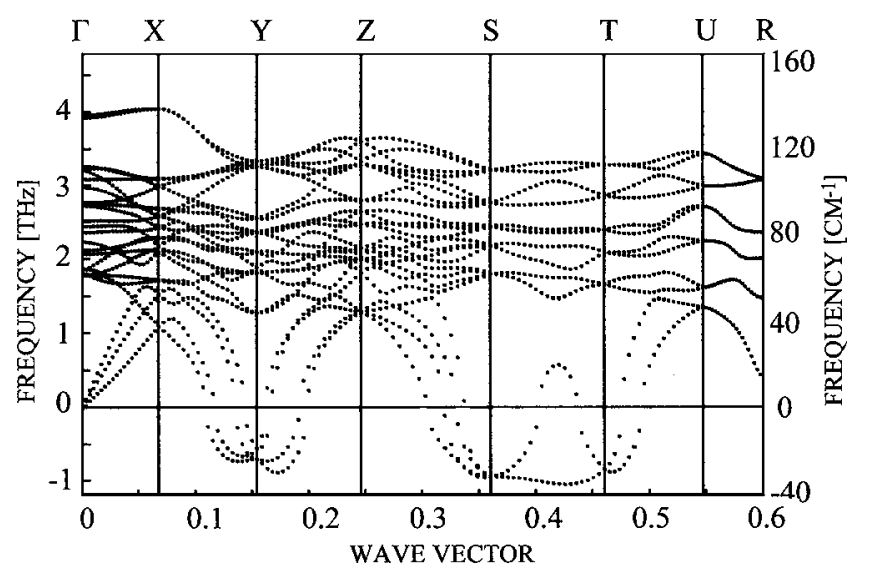

FIG. 2. Dispersion curves in the low-frequency region for $\mathrm{C}_{6} \mathrm{H}_{6}$ using the experimentally determined Ref. 1 unit-cell parameters: $a=7.355 \AA$, $b$ $=9.371 \AA$, and $c=6.700 \AA$ (cell I). The negative values are used to indicate the magnitude of the imaginary frequencies.

\section{RESULTS AND DISCUSSION}

\section{Energy minimization and unit-cell optimization}

The first step in vibrational analysis is the optimization of the crystal structure so that the total energy is a minimum and the forces acting on the atoms are zero. However, optimization of the unit-cell parameters of weakly bound molecular crystals using DFT is not straightforward because long-range attractive interactions due to mutually induced dipoles cannot, in principle, be built into a theory based on one-electron density such as DFT using local-density approximation (LDA or GGA) exchange-correlation functionals. The fact that the dispersive interactions extend over the spatial range from $\sim 3$ to $\sim 8 \AA$ gives rise to a smoothly varying energy variation within the cell that can be considered in terms of a mean field.

This reasoning underlies the correction applied here in which the unit-cell parameters are constrained to experimental (or other) values in order to prevent unphysical cell expansion. For the present type of work, this is the only practical approach to the problem. Where dispersion has been calculated in other cases ${ }^{8,9}$ the approach has been found to work reasonably well. A more direct test of this correction is the calculation of weak rotational potentials for methyl groups, which depend significantly on van der Waals (vdW) interactions and are obtained with a precision of $\sim 90 \%$, see, for example, Ref. 18, which does not include nonlocal, longrange correlation effects such as dispersive interactions. In the present work this is crucial since we will show that rather small changes to the unit-cell parameters have important effects on the lattice dynamics. At worst, this can be conceived as three adjustable parameters: the pressure along each of the crystallographic directions. This means that the calculated variation of properties as a function of pressure will be incorrect by a constant factor that will be rather close to unity, but that the trends will be correct.

The starting model was taken from the most recent crystal structure determination $(4.2 \mathrm{~K}),{ }^{1}$ the atomic positions being relaxed, but with the unit-cell parameters being held constant. A lattice dynamics calculation using the optimized structure resulted in dispersion curves illustrated in Fig. 2. It 


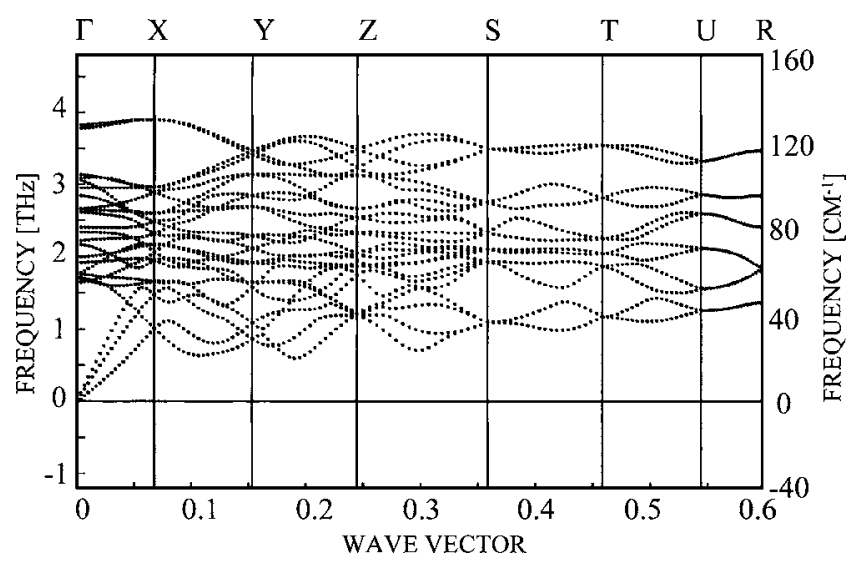

FIG. 3. Dispersion curves in the low-frequency region for $\mathrm{C}_{6} \mathrm{H}_{6}$ using the bigger unit-cell parameters: $a=7.397 \AA, b=9.422 \AA$, and $c=6.737 \AA$ (cell II). Notice the absence of negative (imaginary) values compared with Fig. 2.

is immediately clear from this figure that the acoustic modes and one of the optic modes become imaginary around $Y\left(0, \frac{1}{2}, 0\right), S\left(\frac{1}{2}, \frac{1}{2}, 0\right)$, and $T\left(0, \frac{1}{2}, \frac{1}{2}\right)$. While this may at first seem to be consistent with the proposal that this structure of benzene is only entropically stable, ${ }^{2}$ as we will show later, the lattice-mode INS spectrum calculated with this structure is in poor agreement with that measured.

As alluded to above, there is a small uncertainty in the unit-cell parameters due to the shortcoming of the current DFT method, and consequently we investigated the effects of slight scaling of the unit-cell parameters. A number of calculations were made using larger and smaller unit cells and it transpired that reducing the unit-cell parameters $(0.5 \%$ $2.0 \%$ ) had little effect on the dispersion curves, while increasing the cell parameters by as little as $0.5 \%(\sim 0.04 \AA)$ removed all imaginary frequencies with the exception of some very small values for the acoustic modes at the $\Gamma$ point, $k=0$. The dispersion curves for the low-energy region of the smaller unit cell are illustrated in Fig. 3, and the observed and calculated INS spectra are shown in Fig. 4.

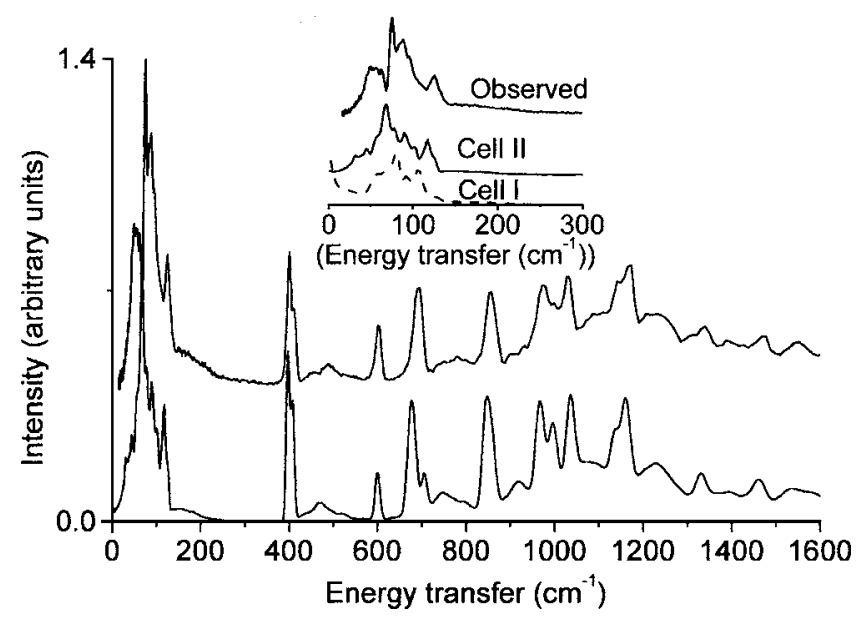

FIG. 4. Observed (upper) and calculated (lower) INS profiles for $\mathrm{C}_{6} \mathrm{H}_{6}$ using the model cell II. Calculation includes multiphonon modes up to five and convolution with an analytical instrumental resolution function. The inset shows the lattice-mode region: observed (upper), cell II (middle), and cell I (lower, dashed).

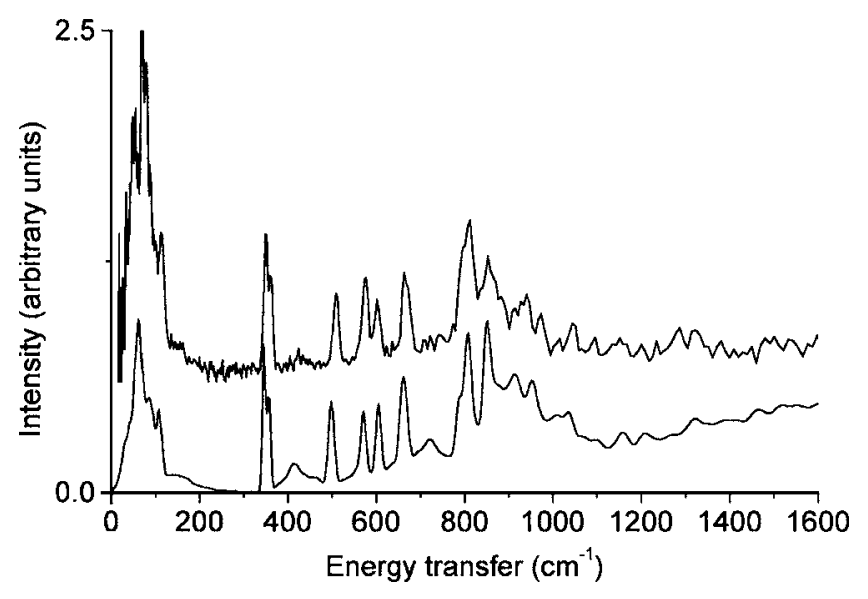

FIG. 5. Observed (upper) and calculated (lower) INS profiles for deuterated benzene, $\mathrm{C}_{6} \mathrm{D}_{6}$, using the model cell II. Calculation includes multiphonon modes up to five and convolution with an analytical instrumental resolution function.

\section{Molecular and lattice vibrations}

We will denote the crystallographically determined unit cell as I and the $0.5 \%$ enlarged cell as II. A comparison of Fig. 2 and 3 reveals considerably different lattice dynamics for such a small change in unit-cell size, without change of symmetry. The inset in Fig. 4 compares the observed and calculated INS spectra in the lattice-mode region for cells I and II, which clearly reveals lost spectral density of the acoustic modes in the experimental cell, with an almost complete absence of intensity in the region around $40 \mathrm{~cm}^{-1}$. In addition the cell-I calculation does not show a Debye-type spectrum in the limit $\omega->0$. While the agreement between the observed spectrum and the spectrum from cell II is not perfect in this region, it is a vast improvement for such a small change in lattice parameters.

Agreement between the observed and calculated spectra of the internal modes is rather good (Fig. 4) and is similar for either unit cell because these modes are much less sensitive to weak intermolecular interactions. The observed and calculated spectra for $\mathrm{C}_{6} \mathrm{D}_{6}$ are shown in Fig. 5, and again it was found that cell II was required to avoid imaginary frequencies and to give good agreement in the low-energy part of the spectrum. Formal assignments of all modes for both isotopomers are given in Table I. Assignments for the calculated frequencies in this table are based on the eigenvectors, and comparison with the experimental values is based on symmetry species where possible, otherwise, best match.

Dispersion for the internal modes is generally less than about $15 \mathrm{~cm}^{-1}$, with notable exception of the $1000 \mathrm{~cm}^{-1}$ region. This spectral region, between 980 and $1010 \mathrm{~cm}^{-1}$, is rather complicated because 12 crystal modes exist, arising from the three molecular modes: ring breathing $\nu_{1}, \mathrm{H}$ wagging $\nu_{5}$, and in-plane ring deformation $\nu_{12}$, these modes (and the proximate $\nu_{17}$ ) being illustrated in Fig. 6. Dispersion of these modes is illustrated in Figs. 7(a) and 7(b) and 8 for cells II and I, respectively. We will first consider the larger cell, II. A comparison of Figs. 7(a) and 7(b) shows that the $\nu_{17}$ wagging modes, between 960 and $980 \mathrm{~cm}^{-1}$ at the zone center, are essentially $\mathrm{H}$-atom displacements over the whole zone. This consistent behavior across the zone is found for 
TABLE I. Vibrational frequencies of crystalline benzene and deuterated benzene at the $\Gamma$ point $(k=0)$. Observed frequencies are given in parentheses. Calculated assignments and their symmetry species are based on the atomic displacements. R and I denote Raman and infrared active modes, respectively. Agreement with the INS data is based on comparison of the observed and calculated spectral profiles (Figs. 4 and 5).

\begin{tabular}{|c|c|c|c|}
\hline $\mathrm{C}_{6} \mathrm{H}_{6}\left(\mathrm{~cm}^{-1}\right)$ & $\mathrm{C}_{6} \mathrm{D}_{6}\left(\mathrm{~cm}^{-1}\right)$ & Assignment (crystal) & Molecular mode \\
\hline-1 & -1 & $B_{2 u}(\mathrm{I})$ & Lattice modes \\
\hline-1 & -1 & $B_{3 u}(\mathrm{I})$ & \\
\hline 0 & 0 & $B_{1 u}(\mathrm{I})$ & \\
\hline $55(57)^{\mathrm{a}}$ & 50 & $A_{g}(\mathrm{R})$ & \\
\hline 56 & 54 & $A_{u}$ & \\
\hline $57(57)^{\mathrm{a}}$ & 53 & $B_{1 g}(\mathrm{R})$ & \\
\hline $59(61)^{\mathrm{a}}(53)^{\mathrm{b}}$ & 5357 & $B_{3 g}(\mathrm{R})+B_{2 u}(\mathrm{I})$ & \\
\hline $64(70)^{\mathrm{b}}$ & 62 & $B_{1 u}(\mathrm{I})$ & \\
\hline 66 & 64 & $A_{u}$ & \\
\hline $72(79)^{\mathrm{a}}$ & 66 & $A_{g}(\mathrm{R})$ & \\
\hline $74(53)^{\mathrm{b}}$ & 71 & $B_{3 u}(\mathrm{I})$ & \\
\hline $78(79)^{\mathrm{a}}$ & 71 & $B_{2 g}(\mathrm{R})$ & \\
\hline $80(100)^{\mathrm{a}}$ & 73 & $B_{1 g}(\mathrm{R})$ & \\
\hline $87(90)^{\mathrm{a}}$ & 79 & $B_{2 g}(\mathrm{R})$ & \\
\hline $89(92)^{\mathrm{a}}(94)^{\mathrm{b}}$ & 8186 & $A_{g}(\mathrm{R})+B_{3 u}(\mathrm{I})$ & \\
\hline $95(92)^{\mathrm{a}}$ & 86 & $B_{3 g}(\mathrm{R})$ & \\
\hline 98 & 95 & $A_{u}$ & \\
\hline $102(85)^{\mathrm{b}}$ & 9395 & $B_{2 g}(\mathrm{R})+B_{1 u}(\mathrm{I})$ & \\
\hline $104(94)^{b}$ & 101 & $B_{2 u}(\mathrm{I})$ & \\
\hline $126(128)^{\mathrm{a}}$ & 115 & $B_{3 g}(\mathrm{R})$ & \\
\hline $127(128)^{\mathrm{a}}$ & 117 & $B_{1 g}(\mathrm{R})$ & \\
\hline 397 & 345 & $B_{2 u}(\mathrm{I})+B_{3 u}(\mathrm{I})$ & $\nu_{16} E_{2 u}$ \\
\hline $399(401)^{\mathrm{c}}$ & $347(353)^{\mathrm{c}}$ & $A_{u}$ & \\
\hline $399(404)^{\mathrm{c}}$ & 348 & $A_{u}+B_{1 u}(\mathrm{I})$ & \\
\hline $402(405)^{\mathrm{c}}$ & 351 & $B_{2 u}(\mathrm{I})$ & \\
\hline 412 & 361 & $B_{3 u}(\mathrm{I})$ & \\
\hline 412 & $361(364)^{\mathrm{c}}$ & $B_{1 u}(\mathrm{I})$ & \\
\hline 597 & 568 & $B_{2 g}(\mathrm{R})$ & $\nu_{6} E_{2 g}$ \\
\hline 598 & 570 & $A_{g}(\mathrm{R})$ & \\
\hline 598 & 571 & $B_{3 g}(\mathrm{R})$ & \\
\hline $600(600)^{\mathrm{d}}$ & 571572 & $A_{g}(\mathrm{R})+B 1_{g}(\mathrm{R})$ & \\
\hline 601 & 571 & $B_{1 g}(\mathrm{R})$ & \\
\hline 602 & 573 & $B_{2 g}(\mathrm{R})$ & \\
\hline $603(606)^{\mathrm{d}}$ & 576 & $B_{3 g}(\mathrm{R})$ & \\
\hline 664 & 489 & $B_{3 u}(\mathrm{I})$ & $\nu_{11} A_{2 u}$ \\
\hline $666(685)^{\mathrm{c}}$ & $490(515)^{\mathrm{c}}$ & $B_{1 u}(\mathrm{I})$ & \\
\hline $691(674)^{c}$ & $507(506)^{\mathrm{c}}$ & $A_{u}$ & \\
\hline $692(690)^{c}$ & $508(525)^{\mathrm{c}}$ & $B_{2 u}(\mathrm{I})$ & \\
\hline 703 & 599 & $B_{3 g}(\mathrm{R})$ & $\nu_{4} B_{2 g}$ \\
\hline 703 & 600 & $B_{1 g}(\mathrm{R})$ & \\
\hline 707 & 609 & $B_{2 g}(\mathrm{R})$ & \\
\hline 708 & 611 & $A_{g}(\mathrm{R})$ & \\
\hline 838 & 653 & $A_{g}(\mathrm{R})$ & $\nu_{10} E_{1 g}$ \\
\hline 839 & 653 & $B_{1 g}(\mathrm{R})$ & \\
\hline 842 & 656 & $A_{g}(\mathrm{R})$ & \\
\hline 847 & 660 & $B_{2 g}(\mathrm{R})$ & \\
\hline 850 & 662 & $B_{3 g}(\mathrm{R})$ & \\
\hline $853(852)^{\mathrm{d}}$ & 665 & $B_{2 g}(\mathrm{R})$ & \\
\hline 866 & 671 & $B_{3 g}(\mathrm{R})$ & \\
\hline 867 & 672 & $B_{1 g}(\mathrm{R})$ & \\
\hline 961 & 784 & $B_{3 u}(\mathrm{I})$ & \\
\hline 962 & 784 & $B_{2 u}(\mathrm{I})$ & $\nu_{17} E_{2 u}$ \\
\hline $963(974)^{\mathrm{c}}$ & $785(795)^{\mathrm{c}}$ & $A_{u}$ & \\
\hline 966 & 788 & $B_{1 u}(\mathrm{I})$ & \\
\hline $967(983)^{c}$ & 786788 & $A_{u}+B_{2 u}(\mathrm{I})$ & \\
\hline 977 & 792 & $B_{3 u}(\mathrm{I})$ & \\
\hline $978(977)^{\mathrm{c}}$ & $792(799)^{\mathrm{c}}$ & $B_{1 u}(\mathrm{I})$ & \\
\hline
\end{tabular}


TABLE I. (Continued.)

\begin{tabular}{|c|c|c|c|}
\hline $\mathrm{C}_{6} \mathrm{H}_{6}\left(\mathrm{~cm}^{-1}\right)$ & $\mathrm{C}_{6} \mathrm{D}_{6}\left(\mathrm{~cm}^{-1}\right)$ & Assignment (crystal) & Molecular mode \\
\hline $988(978)^{\mathrm{d}}$ & 823 & $B_{3 g}(\mathrm{R}) \nu_{5}$ & $\nu_{1} A_{1 g}$ \\
\hline $989(983)^{\mathrm{d}}$ & 823 & $B_{1 g}(\mathrm{R}) \nu_{5}$ & $\nu_{5} B_{2 g}$ \\
\hline 992 & 953 & $B_{3 u}(\mathrm{I}) \nu_{12}$ & $\nu_{12} B_{1 u}$ \\
\hline $992(1010)^{\mathrm{c}}$ & $953(970)^{\mathrm{c}}$ & $B_{1 u}(\mathrm{I}) \nu_{12}$ & \\
\hline 993 & 954 & $B_{2 u}(\mathrm{I}) \nu_{12}$ & \\
\hline $995(991)^{\mathrm{d}}$ & 827950 & $A_{g}(\mathrm{R}) \nu_{1}+B_{1 g}(\mathrm{R}) \nu_{1}$ & \\
\hline $996\left(997^{c}, 1006^{d}\right)$ & $826957(967)^{\mathrm{c}}$ & $B_{2 g}(\mathrm{R}) \nu_{1}+A_{u} \nu_{12}$ & \\
\hline 997 & 952 & $B_{3 g}(\mathrm{R}) \nu_{1}$ & \\
\hline 1003 & 951 & $B_{2 g}(\mathrm{R}) \nu_{5}$ & \\
\hline $1006(1006)^{\mathrm{d}}$ & 952 & $A_{g}(\mathrm{R}) \nu_{5}$ & \\
\hline 1031 & 801 & $B_{2 u}(\mathrm{I})$ & $\nu_{18} E_{1 u}$ \\
\hline 1034 & 803 & $B_{1 u}(\mathrm{I})+B_{3 u}(\mathrm{I})$ & \\
\hline $1035(1035)^{\mathrm{c}}$ & $803(810)^{\mathrm{c}}$ & $A_{u}$ & \\
\hline 1036 & 807 & $A_{u}$ & \\
\hline $1039(1038)^{\mathrm{c}}$ & $808(814)^{\mathrm{c}}$ & $B_{3 u}(\mathrm{I})$ & \\
\hline 1040 & 808 & $B_{1 u}(\mathrm{I})$ & \\
\hline 1041 & 810 & $B_{2 u}(\mathrm{I})$ & \\
\hline 1132 & 809 & $B_{3 u}(\mathrm{I})$ & \\
\hline $1134(1142)^{\mathrm{c}}$ & $810(823)^{\mathrm{c}}$ & $A_{u}$ & $\nu_{15} B_{2 u}$ \\
\hline 1138 & 813 & $B_{1 u}(\mathrm{I})$ & \\
\hline 1142 & 816 & $B_{2 u}(\mathrm{I})$ & \\
\hline 1154 & 844 & $A_{g}(\mathrm{R})$ & $\nu_{9} E_{2 g}$ \\
\hline 1159 & 849 & $B_{1 g}(\mathrm{R})$ & \\
\hline 1159 & 849 & $B_{2 g}(\mathrm{R})$ & \\
\hline 1160 & 849 & $B_{3 g}(\mathrm{R})$ & \\
\hline $1162(1169)^{\mathrm{d}}$ & 850 & $A_{g}(\mathrm{R})$ & \\
\hline $1163(1174)^{\mathrm{d}}$ & 853 & $B_{2 g}(\mathrm{R})$ & \\
\hline $1164(1177)^{d}$ & 852 & $B_{3 g}(\mathrm{R})$ & \\
\hline $1169(1181)^{\mathrm{d}}$ & 857 & $B_{1 g}(\mathrm{R})$ & \\
\hline 1328 & 1036 & $B_{3 g}(\mathrm{R})$ & $\nu_{3} A_{2 g}$ \\
\hline 1330 & 1037 & $A_{g}(\mathrm{R})$ & \\
\hline 1332 & 1039 & $B_{1 g}(\mathrm{R})$ & \\
\hline 1334 & 1040 & $B_{2 g}(\mathrm{R})$ & \\
\hline 1346 & 1324 & $B_{3 u}(\mathrm{I})$ & $\nu_{14} B_{2 u}$ \\
\hline 1348 & 1322 & $B_{1 u}(\mathrm{I})$ & \\
\hline $1348(1312)^{\mathrm{c}}$ & $1325(1286)^{\mathrm{c}}$ & $A_{u}$ & \\
\hline 1349 & 1324 & $B_{2 u}(\mathrm{I})$ & \\
\hline 1459 & 1325 & $B_{1 u}(\mathrm{I})$ & $\nu_{19} E_{1 u}$ \\
\hline 1461 & 1325 & $B_{2 u}(\mathrm{I})$ & \\
\hline $1463(1470)^{\mathrm{c}}$ & $1327(1326)^{\mathrm{c}}$ & $A_{u}$ & \\
\hline 1463 & 1327 & $B_{3 u}(\mathrm{I})$ & \\
\hline $1463(1478)^{\mathrm{c}}$ & 1344 & $B_{2 u}(\mathrm{I})$ & \\
\hline $1465(1475)^{\mathrm{c}}$ & $1343(1329)^{\mathrm{c}}$ & $B_{1 u}(\mathrm{I})$ & \\
\hline 1467 & 1343 & $B_{3 u}(\mathrm{I})$ & \\
\hline 1468 & 1344 & $A_{u}$ & \\
\hline $1588(1585)^{\mathrm{d}}$ & 155115521551 & $A_{g}(\mathrm{R})+B_{2 g}(\mathrm{R})+B_{3 g}(\mathrm{R})$ & $\nu_{8} E_{2 g}$ \\
\hline 1589 & 15521553 & $B_{1 g}(\mathrm{R})+B_{3 g}(\mathrm{R})$ & \\
\hline 1590 & 1555 & $A_{g}(\mathrm{R})$ & \\
\hline 1590 & 1553 & $B_{2 g}(\mathrm{R})$ & \\
\hline 1592 & 1555 & $B_{1 g}(\mathrm{R})$ & \\
\hline 3100 & 2291 & $B_{1 u}(\mathrm{I})+B_{3 u}(\mathrm{I})$ & $\nu_{13} B_{1 u}$ \\
\hline 3102 & 2292 & $A_{u}$ & \\
\hline 3102 & 2293 & $B_{2 u}(\mathrm{I})$ & \\
\hline $3106(3044)^{\mathrm{d}}$ & 2298 & $B_{2 g}(\mathrm{R})$ & $\nu_{7} E_{2 g}$ \\
\hline $3107(3048)^{\mathrm{d}}$ & 22982299 & $A_{g}(\mathrm{R})+B_{1 g}(\mathrm{R})$ & \\
\hline 3107 & 2299 & $B_{3 g}(\mathrm{R})$ & \\
\hline 3111 & 2302 & $B_{2 g}(\mathrm{R})$ & \\
\hline 3111 & 2302 & $A_{g}(\mathrm{R})$ & \\
\hline 3112 & 2303 & $B_{3 g}(\mathrm{R})$ & \\
\hline 3113 & 2304 & $B_{1 g}(\mathrm{R})$ & \\
\hline
\end{tabular}


TABLE I. (Continued.)

\begin{tabular}{llll}
\hline \hline $\mathrm{C}_{6} \mathrm{H}_{6}\left(\mathrm{~cm}^{-1}\right)$ & $\mathrm{C}_{6} \mathrm{D}_{6}\left(\mathrm{~cm}^{-1}\right)$ & Assignment $($ crystal $)$ & Molecular mode \\
\hline 3117 & 2315 & $B_{2 u}(\mathrm{I})$ & $\nu_{20} E_{1 u}$ \\
$3118(3033)^{\mathrm{c}}$ & $2315(2267)^{\mathrm{c}}$ & $A_{u}$ & \\
$3118(3038)^{\mathrm{c}}$ & $2315(2278)^{\mathrm{c}}$ & $B_{1 u}(\mathrm{I})$ & \\
$3119(3088)^{\mathrm{c}}$ & 2316 & $B_{3 u}(\mathrm{I})$ & \\
$3125(3069)^{\mathrm{c}}$ & $2319(2282)^{\mathrm{c}}$ & $B_{2 u}(\mathrm{I})$ & \\
$3125(3092)^{\mathrm{c}}$ & 2320 & $A_{u}$ & \\
3126 & 2320 & $B_{1 u}(\mathrm{I})+B_{3 u}(\mathrm{I})$ & \\
$3128(3061)^{\mathrm{d}}$ & 2327 & $B_{3 g}(\mathrm{R})$ & \\
3130 & 2328 & $B_{1 g}(\mathrm{R})$ & \\
3131 & 2329 & $B_{2 g}(\mathrm{R})$ & \\
3131 & 2329 & $A_{g}(\mathrm{R})$ & \\
\hline \hline
\end{tabular}

${ }^{\mathrm{a}}$ Reference 4.

${ }^{\mathrm{b}}$ Reference 5 .

${ }^{\mathrm{c}}$ Reference 7 .

${ }^{\mathrm{d}}$ Reference 6.

all modes except $\nu_{1}, \nu_{5}$, and $\nu_{12}$, which mix strongly with each other at different wave vectors as is evident from Figs. 7 (a) and 7(b) between 980 and $1010 \mathrm{~cm}^{-1}$. Away from the zone center, the displacements in these modes are clearly a mixture of the formal molecular modes: ring breathing (symmetric and antisymmetric) with the out-of-plane wagging modes. The situation for the smaller cell, I, is markedly different [Figs. 8(a) and 8(b)]. Not only is the dispersion in this region more pronounced but here the higher-frequency components of $\nu_{17}$ also mix with $\nu_{1}, \nu_{5}$, and $\nu_{12}$ away from the zone center.

This mixing of out-of-plane H-wagging modes with inplane ring breathing modes away from the zone center suggests a significant intermolecular interaction between the $\mathrm{H}$ atoms of one molecule and the aromatic core of the neighboring molecule (see below). The molecular center-of-mass displacement of these modes also varies across the zone due to the coupling of these internal modes with the lattice modes.
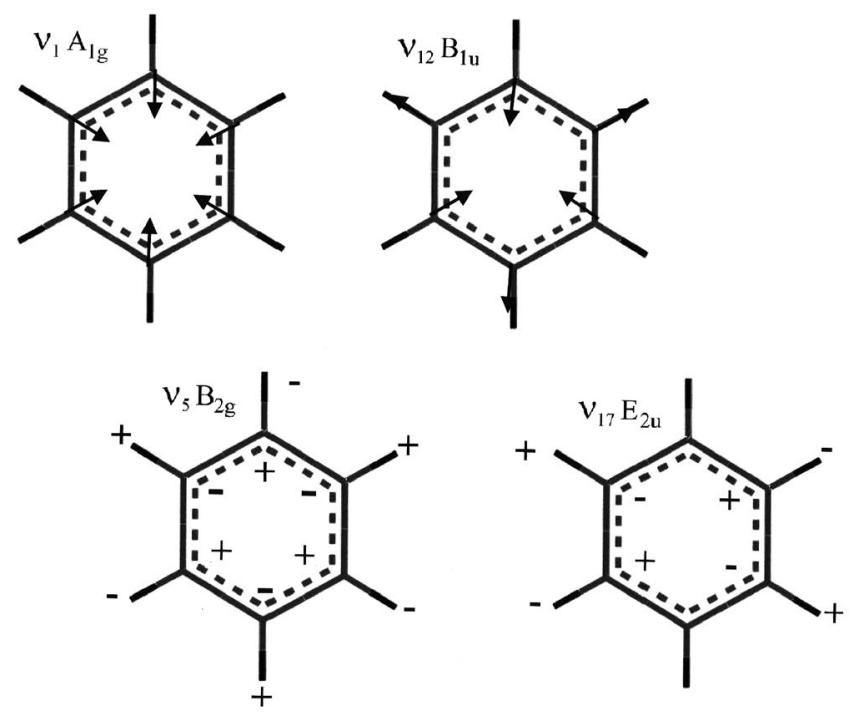

FIG. 6. Schematic illustration of the atomic displacements in the molecular modes that arise in the $960-1010 \mathrm{~cm}^{-1}$ spectral region ( $\Gamma$ point).

\section{Intermolecular interactions}

The crystal structure of benzene illustrated in Fig. 1 is conveniently regarded as composed of layers of molecules stacked along the long $b$ axis, the molecules in each $a c$ layer are tilted by about $38^{\circ}$ to $b$. Inspection of the crystal structure reveals the three types of interaction that are illustrated in Fig. 9. The interactions labeled A and B are between the layers, while the layers themselves are held together by in-
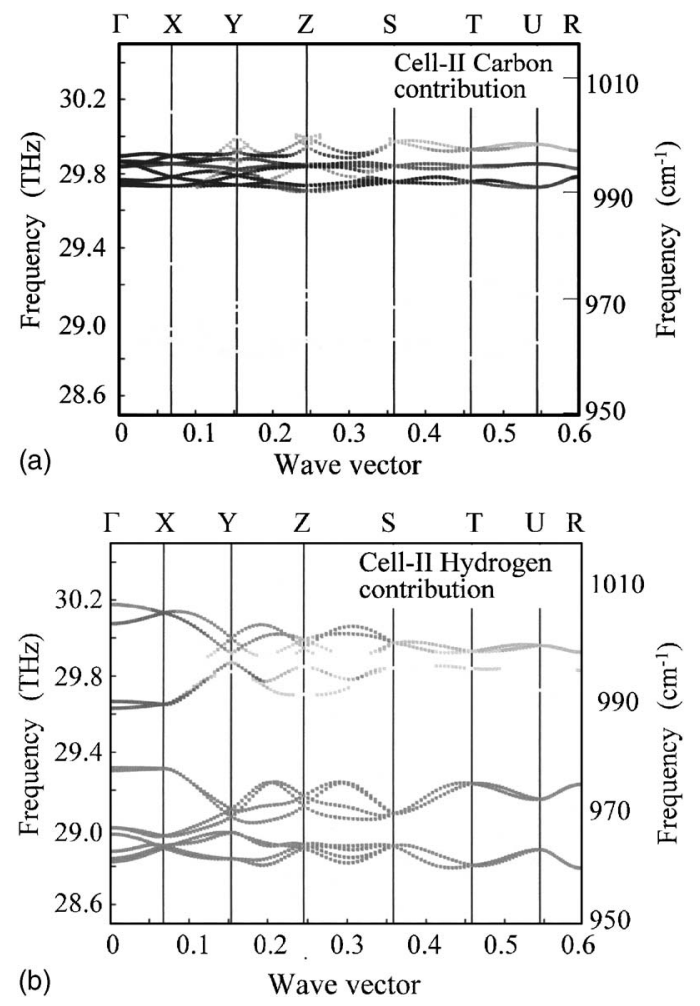

FIG. 7. Mixing of formal molecular modes with each other and lattice modes across the zone. The behavior is not seen in other spectral regions. (a) Dispersion curves for cell II in the regions of $\nu_{17}$ (between 961 and $\left.978 \mathrm{~cm}^{-1}\right), \nu_{5}\left(\sim 990\right.$ and $\left.\sim 1005 \mathrm{~cm}^{-1}\right), \nu_{12}\left(\sim 992\right.$ and $\left.996 \mathrm{~cm}^{-1}\right)$, and $\nu_{1}\left(\sim 996 \mathrm{~cm}^{-1}\right)$, these frequencies being at the $\Gamma$ point, $k=0$. The density of the lines (gray scale) reflects the relative amplitude of the $\mathrm{C}$-atom displacements. (b) Same as (a), but the intensity of the lines (gray scale) represents the relative amplitude of the $\mathrm{H}$-atom displacements. 


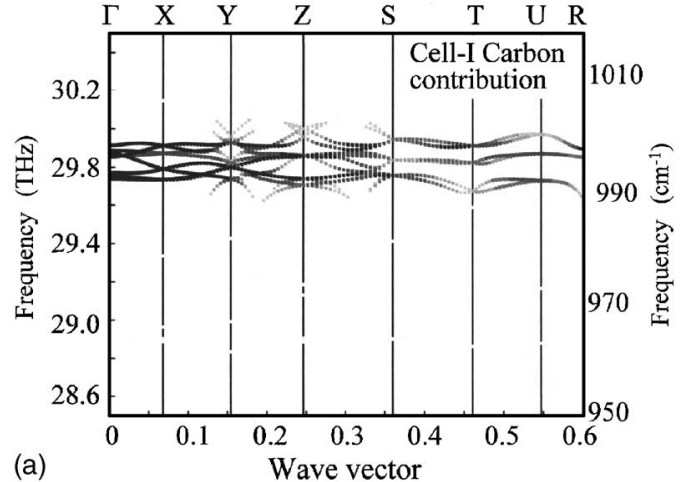

(a)

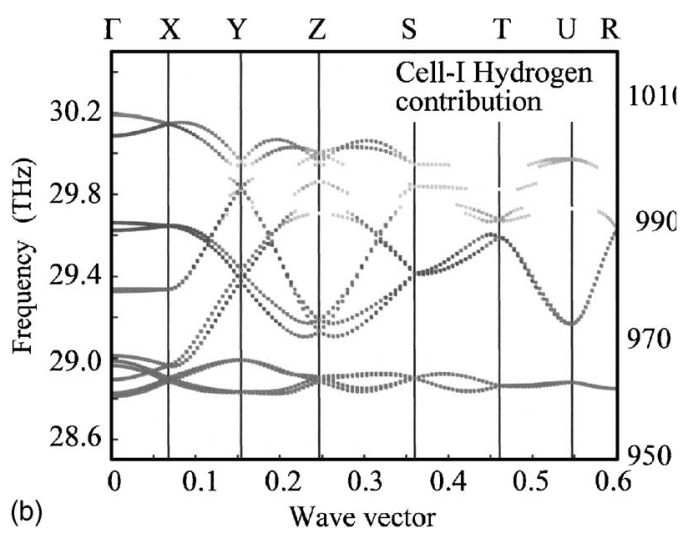

FIG. 8. (a) Dispersion curves for cell II in the regions of $\nu_{17}, \nu_{5}, \nu_{12}$, and $\nu_{1}$, to be compared with Fig. 7(a). The intensity of the lines (gray scale) reflects the relative amplitude of the C-atom displacements. (b) Same as (a), but the intensity of the lines (gray scale) represents the relative amplitude of the $\mathrm{H}$-atom displacements.

teraction $\mathrm{D}$, and a more unusual type of interaction between the $\mathrm{H}$ atoms and the aromatic rings of neighboring molecules, labeled C. In this interaction, the distance from the $\mathrm{H}$ atom to each $\mathrm{C}$ atom of the neighboring ring is almost the same, varying from 3.015 to $3.061 \AA$, the distance from the $\mathrm{H}$ atom to the center of the ring (labeled E) being only 2.701 $\AA$.

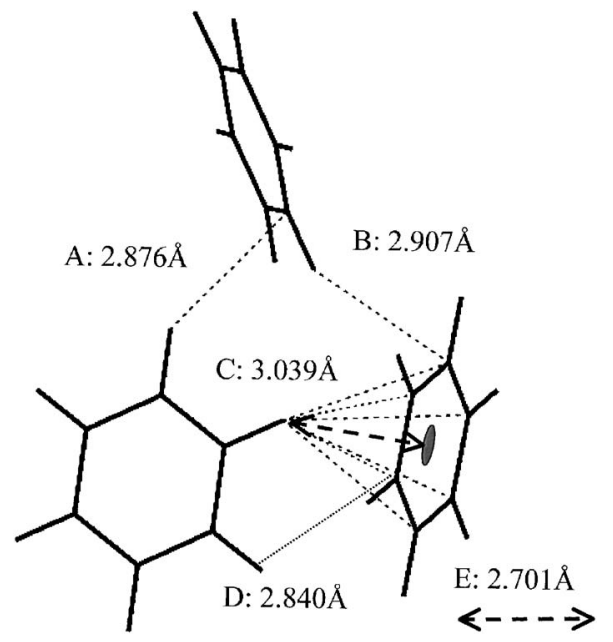

FIG. 9. Illustration of the three major intermolecular interactions and their distances. The oval represents the center of the aromatic ring, the intermolecular distance to the nearest neighbor being E, $2.701 \AA$. The orientation of this fragment is similar to that of the unit cell in Fig. 1.
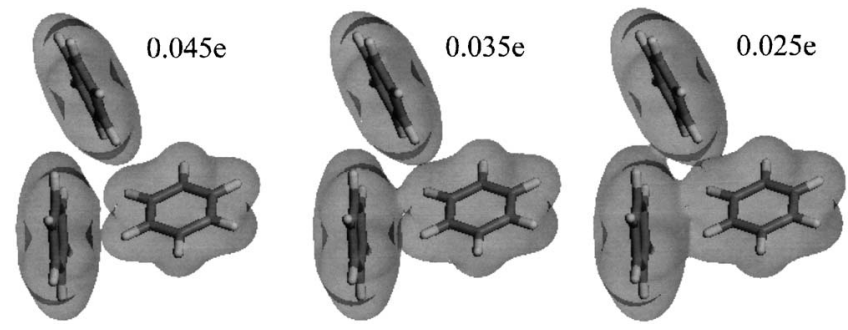

FIG. 10. Development of the electron density for interactions within the $a c$ plane (lower molecules) and between planes (upper molecule), as the isosurface is decreased from $0.045 e$ to $0.025 e$. Notice the large change in the $\mathrm{H}$-aromatic overlap between $0.035 e$ and $0.025 e$.

These interactions are examined in more detail in Fig. 10, where the electron-density isosurface has been calculated at three different values. At the isosurface $0.045 e$ the beginning of interaction type $\mathrm{C}$ can be seen between the two lower molecules, but the $\mathrm{H}$-aromatic link has not actually been made. Looking at lower electron density, 0.035, the interaction type $\mathrm{D}$ is established but there is no actual link between the upper and lower molecules, and the $\mathrm{H}$-aromatic link is still missing. Finally, at the isosurface $0.025 e$, all links are established, but it is interesting to note that the greatest overlap is by a combination of interactions $\mathrm{C}$ and $\mathrm{D}$, which effectively merge the densities of the two lower molecules. The picture that emerges from this electron-density figure is of molecules that are held together in layers (lower molecules) by a quite strong interaction plus the H-aromatic interactions. The layers are linked to each other by weaker interactions. This picture is broadly consistent with the pattern of acoustic modes in Fig. 2 and 3, where the lowestenergy mode is translation along $Y$, while the corresponding modes for $X$ and $Z$ are generally about $50 \%$ higher.

The anisotropy of the mean-square displacements, collected in Table II, is also in agreement with this picture. Considering the $\mathrm{H}$ atom involved in the $\mathrm{H}$-aromatic interaction, $\mathrm{C}$, displacement perpendicular to the approximate bond direction is noticeably larger than that in the $x-z$ plane. Similarly, H-atom interactions involved in connecting neighboring planes of molecules, along $y$, show less overall displacement in this direction. The overall isotropic values are in reasonable agreement with those measured crystallographically. ${ }^{1}$

TABLE II. Calculated mean-square displacements for the crystallographically distinct atoms. The interaction types, $a-d$, are illustrated in Fig. 9. Figures in parentheses are for the $\mathrm{H}$ atom after subtraction of the $\mathrm{C}$ atom displacement.

\begin{tabular}{lllll}
\hline \hline $\begin{array}{l}\text { Atom interaction } \\
\text { Atom interaction }\end{array}$ & MSD- $x\left(\AA^{2}\right)$ & MSD- $y\left(\AA^{2}\right)$ & MSD $-z\left(\AA^{2}\right)$ & Isotropic $\left(\AA^{2}\right)$ \\
\hline $\mathrm{H} a$ & $0.74(0.52)$ & $0.71(0.54)$ & $0.73(0.53)$ & $0.73(0.53)$ \\
$\mathrm{H} c$ & $0.70(0.51)$ & $0.78(0.57)$ & $0.75(0.54)$ & $0.74(0.54)$ \\
$\mathrm{H} d$ & $0.74(0.52)$ & $0.61(0.50)$ & $0.79(0.55)$ & $0.71(0.52)$ \\
$\mathrm{C} a$ & 0.22 & 0.17 & 0.20 & 0.20 \\
$\mathrm{C} c$ & 0.19 & 0.21 & 0.21 & 0.20 \\
$\mathrm{C} d$ & 0.22 & 0.11 & 0.24 & 0.19 \\
\hline \hline
\end{tabular}




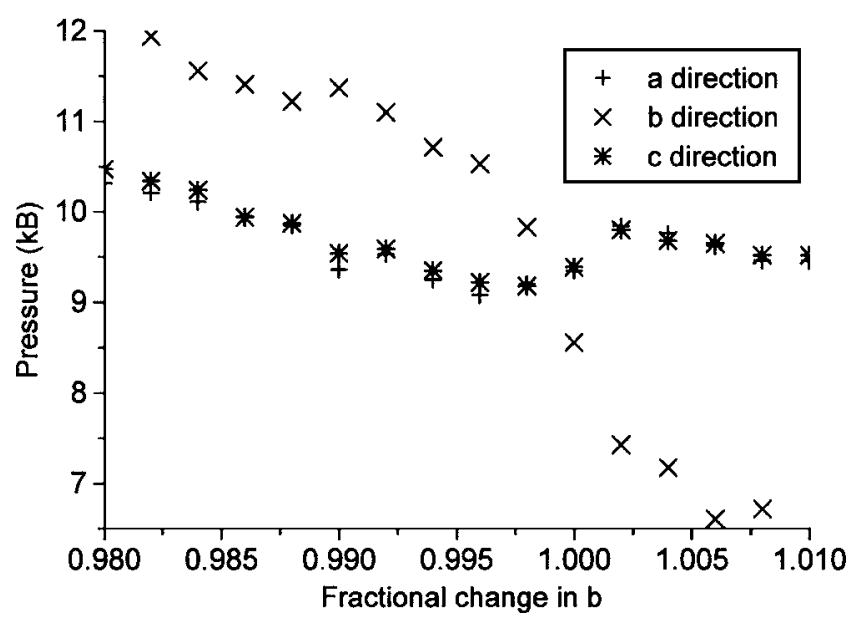

FIG. 11. Plot of calculated pressure in cell II along each direction as a function of the fractional change in the lattice parameter $b$ (for $1.000 b$ $=6.737 \AA$ ). The atomic positions were relaxed to the energy minimum for each value of $c$.

\section{Auxetic effect of pressure along $b$ direction}

Intuitively we would expect the stronger interactions in the $a c$ plane to be more sensitive to changes in the unit-cell size, but this is clearly not the case.

In order to proceed we have investigated the structural consequences on progressively changing the unit-cell $b$ parameter. Figure 11 shows the results of these calculations starting from the parameters of cell II, and it can be seen that within the range on the $b$ parameters of cells I and II, there is a marked hardening of the cell along this direction. Perhaps more surprising is that in the range from 1.002 to 0.996 , the pressure along $b$ increases by $3.1 \mathrm{kbars}$, while the pressure along both $a$ and $c$ reduces by 0.7 kbars. In terms of cell parameters, shortening $b$ leads to compression along $a$ and $c$, giving a negative Poisson's ratio or auxetic behavior. This behavior can be understood by considering Figs. 1 and 9 . The structural differences at the points 0.99 and 1.01 in Fig. 11 correspond to changes in C and D (Fig. 9), of only 0.002 $\AA$ this being consistent with the greater overlap of electron density shown on the right of Fig. 10. In contrast, distances $\mathrm{A}$ and $\mathrm{B}$, between neighboring layers, decrease by 0.013 and $0.023 \AA$ for compressing the $b$ direction from 1.01 to 1.00 , but then increase by 0.040 and $0.016 \AA$, respectively, when compressing the $b$ direction further to 0.99 . In order to establish the consistency of this effect we have also calculated the consequences of a $1 \%$ elongation of the cell along $a$. This leads to decreases in pressure along $a$ and $c$ of 1.9 and 1.2 kbars, respectively, but an increase of 0.9 kbars along $b$, this being entirely consistent with the results obtained above.

Without going into the fine detail of the rather small molecular reorientations, the basic scheme is as follows. It is important to notice that interactions A and B also have components in the $a c$ plane so that initially, as the lattice is compressed along $b$, interactions $\mathrm{A}$ and $\mathrm{B}$ increase pulling molecules in the $a c$ plane together, reducing the pressure in this plane. Further compression (below 0.998) forces A and $\mathrm{B}$ interactions beyond their optimum, allowing relaxation in the $x z$ plane.
This pattern of interactions accounts for the high sensitivity of the acoustic modes at points $Y\left(0, \frac{1}{2}, 0\right), S\left(\frac{1}{2}, \frac{1}{2}, 0\right)$, and $T\left(0, \frac{1}{2}, \frac{1}{2}\right)$ in the dispersion curves shown in Figs. 2 and 3. The $b$ parameter of cell I corresponds to 0.995 in Fig. 11, where A and B interactions are not optimal, and hence at some points in the Brillouin zone $y$ displacements lead to an overall drop in the energy, and hence imaginary frequencies. When A and B interactions are near optimal, all frequencies are real (apart from a very small error at $k=0$ for the acoustic modes), as seen in Fig. 3. This change in A and B interactions not only accounts for the changes in the dispersion of the lattice modes between cells I and II (Figs. 2 and 3) but also for the surprisingly large changes in the internal-mode dispersion of the $\mathrm{H}$-wagging modes $\nu_{5}$ and $\nu_{17}$. Inspection of Figs. 1 and 6 reveals that all modes that wag the interlayer hydrogens will strongly modulate A and B (Fig. 9).

\section{CONCLUSIONS}

DFT calculations are now sufficiently rapid and accurate to allow the vibrational dynamics of crystals, such as benzene, and to verify these calculations against experimental spectroscopies. The principle difficulty is the uncertainty in dispersion energy, but this can be overcome by using pressure to constrain the lattice to the experimental values, effectively preventing physically unrealistic lattice expansion. In the case of benzene, however, it is found that very small changes in unit-cell dimensions have a dramatic effect on the lattice dynamics and an expansion of only $0.5 \%$ above the experimentally determined values takes the cell from an unstable to a stable state. Although this leaves a small unknown scalar in the pressure, it is clear that the phonon dispersion and the dispersion of some of the internal modes depend crucially on small changes to the lattice parameters.

The net interactions holding the molecules together in layers are stronger than those holding neighboring layers together. Because some interactions play both roles, forcing the layers together can increase the net interaction within the layers leading to a "contraction" of the layer. Changes in these interactions are entirely consistent with the sensitivity of the lattice modes and molecular vibrations to small changes in the unit-cell size. Constraining the unit-cell parameters to values close to those experimentally determined is effectively a correction of the DFT method to take account of dispersive interactions. This has the consequence of introducing an offset in the pressure of about $10 \mathrm{kbars}$, as seen in Fig. 11. This will have some small effect on the relative values at which the auxetic effect occurs, and it would be interesting to see if the predicted auxetic effect could be observed experimentally.

Dispersion of internal modes is normally only important where strong hydrogen bonding interactions are involved. In the present case there is significant intermolecular coupling of wagging and breathing modes of the aromatic ring that can be seen as Davidov splitting at the zone center, but which couples strongly to optic and acoustic phonons away from the zone center causing extensive mixing. This interaction is considerably stronger than would be suggested by an analysis of the optical spectra alone. 
${ }^{1}$ W. I. F. David, R. M. Ibberson, G. A. Jeffrey, and J. R. Ruble, Physica B 180 \& 181, 597 (1992).

${ }^{2}$ P. Raiteri, R. Martoňák, and M. Parrinello, Angew. Chem., Int. Ed. 44, 2 (2005).

${ }^{3}$ R. Martoňák, A. Laio, M. Bernasconi, C. Ceriani, P. Raiteri, F. Zipoli, and M. Parrinello, Z. Kristallogr. 220, 489 (2005).

${ }^{4}$ H. Bonadeo, M. P. Marzocchi, E. Castellucci, and S. Califano, J. Chem. Phys. 57, 4299 (1972).

${ }^{5}$ G. Taddei, H. Bonadeo, M. P. Marzocchi, and S. Califano, J. Chem. Phys. 58, 966 (1973).

${ }^{6}$ M. M. Thiéry and J. M. Léger, J. Chem. Phys. 89, 4255 (1988).

${ }^{7}$ M. M. Thiéry, J. M. Besson, and J. L. Bribes, J. Chem. Phys. 96, 2633 (1992).

${ }^{8}$ M. R. Johnson and H. P. Trommsdorff, Chem. Phys. Lett. 364, 34-38 (2002).

${ }^{9}$ J. Stride, M. Adams, and M. R. Johnson, Chem. Phys. 317, 143 (2005).

${ }^{10}$ L. van Eijck, M. R. Johnson, and G. J. Kearley, J. Phys. Chem. A 107,
$8980(2003)$

${ }^{11}$ M. Montejo, A. Navarro, G. J. Kearley, J. Vazquez, and J. J. LopezGonzalez, J. Am. Chem. Soc. 126, 15087 (2004).

${ }^{12}$ M. R. Johnson, K. Parlinski, I. Natkaniec, and B. Hudson, Chem. Phys. 53, 291 (2003).

${ }^{13}$ P. Hermet, J.-L. Bantignies, A. Rahmani, J.-L. Sauvajol, M. R. Johnson, and F. Serein, J. Phys.: Condens. Matter 16, 7385 (2004).

${ }^{14}$ P. Hermet, J.-L. Bantignies, A. Rahmani, J.-L. Sauvajol, and M. R. Johnson, J. Phys. Chem. A 109, 4202 (2005).

${ }^{15}$ P. C. H. Mitchell, S. F. Parker, A. J. Ramirez-Cuesta, and J. Tomkinson, Vibrational Spectroscopy with Neutrons (World Scientific, NJ, 2005).

${ }^{16}$ G. Kresse and J. Furthmüller, software VASP, Vienna, 1999; G. Kresse, Phys. Rev. B 54, 169 (1996); Comput. Mater. Sci. 6, 15 (1996).

${ }^{17}$ K. Parlinski, AIP Conf. Proc. 479, 121 (1998).

${ }^{18}$ M. R. Johnson, M. Prager, H. Grimm, M. A. Neumann, G. J. Kearley, and C. C. Wilson, Chem. Phys. 244, 49 (1999). 